\title{
BER and PAPR Analysis of DFT Precoded OFDM Multilayer IDMA (P-OFDM ML IDMA) Signals
}

\author{
Mohamed A. Aboul-Dahab, Esam A. A. A. Hagras, and Ahmad A. Elhaseeb
}

\begin{abstract}
In this paper, Discrete Fourier Transform Precoded Orthogonal Frequency Division Multiplexing Multilayer Interleave Division Multiple Access (DFT P OFDM ML IDMA) communication system has been proposed. Chip by Chip (CBC) iterative receiver is used to recover the data in each layer. Also, different precoding techniques has been applied with and without clipping in the proposed P OFDM ML IDMA. Simulation results confirm that the DFT precoder provides better PAPR reduction compared with other precoded methods with and without clipping. BER and PAPR simulation results shows that the proposed DFT P OFDM ML IDMA brings about $7 \mathrm{~dB}$ PAPR reduction and $0.5 \mathrm{~dB}$ BER improvement compred with OFDM ML IDMA. The Simulations for the proposed DFT P OFDM ML IDMA with 2 - 6 layers shows that the variation is 3.4 to $8.2 \mathrm{~dB}$ for the PAPR and 7.8 to $8.7 \mathrm{~dB}$ for the BER. So, the adaptive modulation can be simply realized by the proposed DFT P OFDM ML IDMA.
\end{abstract}

Index Terms - PAPR, DFT, precoding, OFDM and IDMA.

\section{INTRODUCTION}

Interleave-Division Multiple-Access (IDMA) was recently investigated. IDMA uses different chiplevel interleavers to distinguish different users, all adopting the same spreading sequence. The key principle of IDMA systems is that the chip-level interleavers should be different for different users. In addition, a low-cost $\mathrm{CBC}$ iterative detection can be utilized in the IDMA systems [1], [2]. Orthogonal Frequency Division Multiplexing (OFDM) is a multicarrier transmission scheme that has become the technology choice for next generation wireless and wire line digital communication systems [3].

IDMA can be combined with OFDM (OFDM-IDMA) integrates the advantages of both OFDM and IDMA in order to mitigate the intersymbol interference (ISI) and suppress the multiple access interference (MAI) simultaneously [4], [5]. OFDM-IDMA suffers PAPR problem. The transmitted signal of OFDM-IDMA has high PAPR, which may degrade the efficiency of power amplifier of the transmitters.

A number of PAPR reduction schemes have been proposed for OFDM systems, such as clipping [6], partial transmit sequence method (PTS) [7], and selected mapping method (SLM) [8]. In [9],clipping is adopted in OFDMIDMA systems for PAPR reduction but interference from clipping noise will reduce BER performance.

To alleviate the above problem, precoding based

Manuscript received March 1, 2013; revised April 16, 2013.

The authors are with the Elec. and com. Dept., Faculty of Engineering, Arab Academy for Science and Technology and Maritime Transport, Cairo, Egypt (e-mail: mdahab@aast.edu, esamhagras_2006@yahoo.com, dgdfsdf@yahoo.com). techniques, however, show great promise as they are simple linear techniques to implement without the need of any side information. Precoding improves PAPR without increasing much complexity and without destroying the orthogonality between subcarriers. The precoding also improves the BER in comparison to normal OFDM system because of diversity gain obtained due to the spreading of data symbol among more than one subcarrier.

In this paper, we present DFT precoder for clipped and Unclipped OFDM ML IDMA signals to reduce PAPR. Performance comparison is made with other transformbased precoders, namely Discrete Hartley Transform (DHT) and Zadoff-Chu Transform (ZCT) [7], [10].

This paper is organized as follows: Section II presents the proposed DFT P-OFDM ML IDMA system adopted in this paper; in Section III, computer simulation results are given and the system performance is also analyzed; the conclusions are given in Section IV.

\section{PRoposed DFT PC-OFDM ML IDMA}

\section{A. Transmitter}

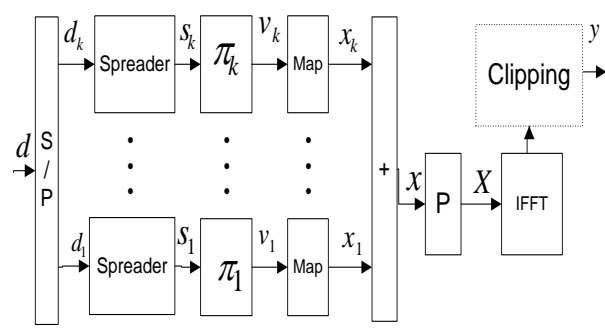

Fig. 1. Proposed DFT P-OFDM ML IDMA transmitter

The transmitter based on OFDM ML IDMA with $K$ layers is shown in Fig. 1 binary data sequence $d$ is first partitioned into $K$ subsequences. For $k$-layer, the data sequence $d_{k}$ is spread using a length-S spreading sequence $\boldsymbol{S}_{k}$.we write the chip sequence obtained after spreading as $\boldsymbol{S}_{k}=\left[s_{k}(1), \ldots, s_{k}(j), \ldots, s_{k}(J)\right]^{T}$, where $\mathrm{J}$ is the block length. Then $\boldsymbol{S}_{k}$ is permutated by an interleaver $\boldsymbol{\pi}_{k}$, yielding $\boldsymbol{v}_{k}$ $=\left[v_{k}(1), \ldots, v_{k}(j), \ldots, v_{k}(J)\right]^{T}$ After interleaving, the sequence $\boldsymbol{v}_{k}$ is modulated to a sequence $x_{k}$ by binary phase shift keying (BPSK) mapping.Then all the data of $K$ layers are linearly superimposed to transmission. The output signal $x$ is a linear superposition of independently symbol in (1).

$$
x(j)=\sum_{k=1}^{K} x_{k}(j) \quad j=0,1, \ldots, J-1
$$

After ML IDMA signal processing, $x$ is passed to DFT precoder $\mathrm{P}$ yielding $X$ in (2): 


$$
X(v)=\sum_{m=0}^{M-1} x(m) \cdot e^{-j 2 \pi m v / M}, v=0,1 \ldots M-1
$$

which have the same size as IFFT is used as a precoded spreading code. Thus, the OFDM system becomes equivalent to the Single Carrier (SC) system because the DFT and IDFT operations virtually cancel each other [11].

In this case, the transmit signal will have the same PAPR as in a single-carrier system which results in improvement in PAPR. We assume that the size of DFT is equal to the block length, namely $M=J$. The transmitted time domain channel symbol that is obtained from the inverse DFT (IDFT) of $\mathrm{X}$ is:

$$
y(n)=\frac{1}{N} \sum_{l=0}^{N-1} X(l) \cdot e^{j 2 \pi n l / N}, n=0,1 \ldots . N-1
$$

\section{B. Receiver}

The received signal can be written as:

$$
R=h \cdot y+n
$$

where $n$ is additive white Gaussian noise (AWGN) with variance $\sigma^{2}$. We assume that the channel gains $h$ are independent, identically distributed and perfectly known at transmitter and receiver. We consider the AWGN channel. The received data is first implemented by FFT and the IDFT precoder. For the sake of attaining the best possible performance, the structure of CBC iterative multiple user detection of IDMA is shown in Fig. 2.

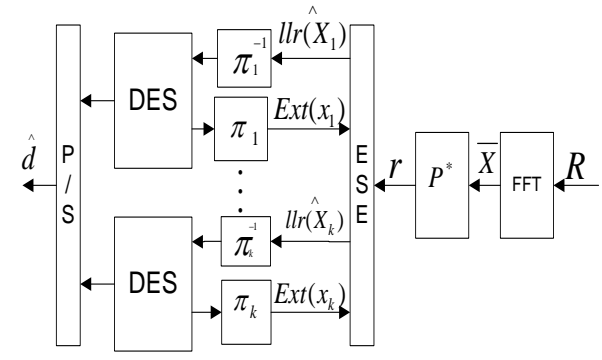

Fig. 2. Proposed DFT P-OFDM ML IDMA receiver

This structure consists of a soft-in-soft-out (SISO) Elementary Signal Estimator (ESE) and a bank of $K$ individual SISO Decoding for the de-spreading operation (DES), where the soft information exchanged between the receiver components is constituted by the extrinsic LogLikelihood Ratios (LLRs).

We adopt the low complexity ESE algorithm derived in [11], [12]. This algorithm is derived through Gaussian approximation based on the assumption that the interfering symbols are independent of each other. This assumption is reasonable when the block is long enough because of the layer-specific random interleavers.

$$
\bar{X}(v)=\sum_{n=0}^{N-1} R(n) e^{-j 2 \pi v n / N} v=0,1, \ldots, N-1
$$

Then implements De-precoding using IDFT, producing $r$

$$
r(v)=\frac{1}{M} \sum_{m=0}^{M-1} \bar{X}(m) e^{j 2 \pi v m / M} v=0,1, \ldots, M-1
$$

In the process of IDMA detection, we reformulate the algorithm for BPSK signaling as:

$$
\begin{aligned}
& r_{k}=h_{k} \sum_{m=0}^{M-1} x_{m}+n_{k} \\
& r_{k}=h_{k} x_{m}+\zeta_{k}
\end{aligned}
$$

where $\zeta_{k}$ is the interference in $r_{k}$ with respect to layer $m$.

$$
\zeta_{k} \equiv r_{k}-h_{k} x_{m}=h_{k} \sum_{m,=0, m, \neq m}^{M-1} x_{m^{\prime}}+n_{k}
$$

From the central limit theorem $\zeta_{k}$ can be approximated as Gaussian variable, and $r_{k}$ can be characterized by conditional Gaussian probability function, e.g. BPSK is adopted $x_{k} \in\{+1,-1\}$.

$p\left(r_{k} \mid x_{m}= \pm 1\right)=\frac{1}{\sqrt{2 \pi v a r\left(\zeta_{k}\right)}} \exp \left[-\frac{\left(r_{k}-\left( \pm h_{k}+E\left(\zeta_{k}\right)\right)\right)^{2}}{2 \operatorname{var}\left(\zeta_{k}\right)}\right]$

where $E(*)$ and $\operatorname{Var}(*)$ are the mean and variance functions, respectively. The output Log Likelihood Rate $(L L R)$ of $\hat{X}$ can be expressed as:

$$
\operatorname{ll}\left(\hat{X}_{m}\right)=\log \left[\frac{p\left(r_{k} \mid x_{m}=+1\right)}{p\left(r_{k} \mid x_{m}=-1\right)}\right]=2 h_{k} \frac{r_{k}-E\left(\zeta_{k}\right)}{\operatorname{var}\left(\zeta_{k}\right)}
$$

where

$$
\begin{gathered}
E\left(\zeta_{k}\right)=E\left(h_{k} \sum_{m^{\prime}=0, m^{\prime} \neq m}^{M-1} x_{m^{\prime}}+n_{k}\right) \\
=h_{k} \sum_{m,=0, m, \neq m}^{M-1} E\left(x_{m}\right) \\
=E\left(r_{k}-h_{k} E\left(x_{m}\right)\right) \\
\operatorname{var}\left(\zeta_{k}\right)=\left|h_{k}\right|^{2} \sum_{k^{\prime}=1}^{K} \operatorname{Var}\left(x_{m}\right)+\sigma^{2} \\
=\operatorname{Var}\left(r_{k}\right)-\left|h_{k}\right|^{2} \operatorname{Var}\left(x_{m}\right) \\
\operatorname{Ext}\left(x_{m}\right)=\log \left[\frac{p\left(x_{m}=+1\right)}{p\left(x_{m}=+1\right)}\right]
\end{gathered}
$$

$E\left(x_{m}\right), \operatorname{Var}\left(x_{m}\right)$ both are determined by the input extrinsic information $\operatorname{Ext}\left(x_{m}\right)$ defined in (14), given by reinterleaver module.

$$
\begin{gathered}
E\left(x_{m}\right)=\tanh \left(\frac{\operatorname{Ext}\left(x_{m}\right)}{2}\right) \\
\operatorname{Var}\left(x_{m}\right)=1-\left(E\left(x_{m}\right)\right)^{2}
\end{gathered}
$$

Thus we can analyze and detect the signal of each layer through the mean and variance of the interlayer interference. And then the updated extrinsic LLR from ESE function goes through the layer-specific deinterleaver and gets into the DESs iteratively. After the last iteration, the DESs produce hard decisions towards information bits in each layer separately. Finally, the decision bits of each layer are parallel-to-serial converted to obtain the recovered data sequence.

\section{Simulation Results}

In this subsection, simulations are carried out to evaluate the performance of P-OFDM ML IDMA system with and without clipping. In the simulations, the BPSK modulation and AWGN channel are employed. The spreading code length is $S=8$, and the spreading sequence $\{+1,-1,+1,-1 \ldots\}$ is adopted for all layers and the subcarriers number is $N=128$ and the layers number is $L=2$, the number of iterations is 3 .

Fig. 3 Shows the PARR comparisons of clipped OFDM ML 
IDMA system at CRs $(0.9,1,2)$ which improve PAPR performance but as shown in Fig. 4 the BER performance is degraded by $4 \mathrm{~dB}$.

Fig. 5 shows the CCDF comparisons of the proposed DFT P-OFDM ML IDMA system (without clipping) which are better than original OFDM ML IDMA, ZCT P-OFDM ML IDMA and DHT P-OFDM ML IDM, by about $7 \mathrm{~dB}$, $3 \mathrm{~dB}, 6 \mathrm{~dB}$ respectively.

As shown in Fig. 6 the proposed system (without clipping) using DFT precoder improves the BER performance by about $0.3 \mathrm{~dB}$ compared with original system.

Fig. 7 shows that the PAPR for both DFT P OFDM ML IDMA with and without clipping. It is shown that they have the same PAPR. The proposed DFT precoder improves the BER performance by about $0.3 \mathrm{~dB}$ compared with clipped system as shown in Fig. 8.

Fig. 9 shows the PAPR comparisons for different layers $=2,3,4,5$ and 6 where the proposed DFT precoder system using 6 layers is better than original system using 2 layers by about $2 \mathrm{~dB}$ in addition to keep the BER performance as shown in Fig. 10.

The final simulated results for the proposal DFT $P$ OFDM ML IDMA system is shown in Table I and II.

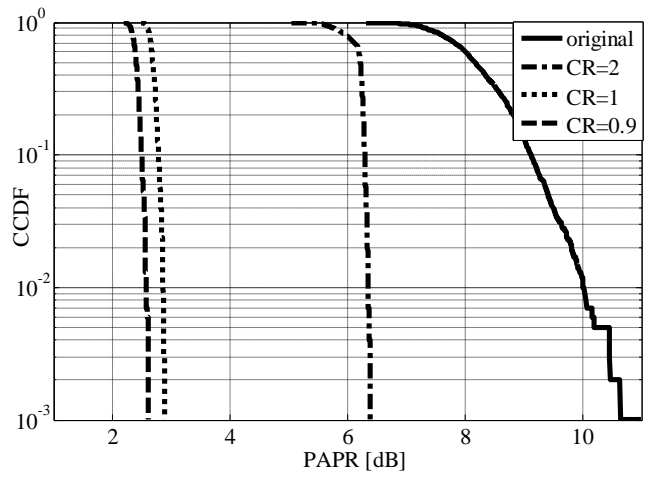

Fig. 3. PAPR of clipped OFDM ML IDMA

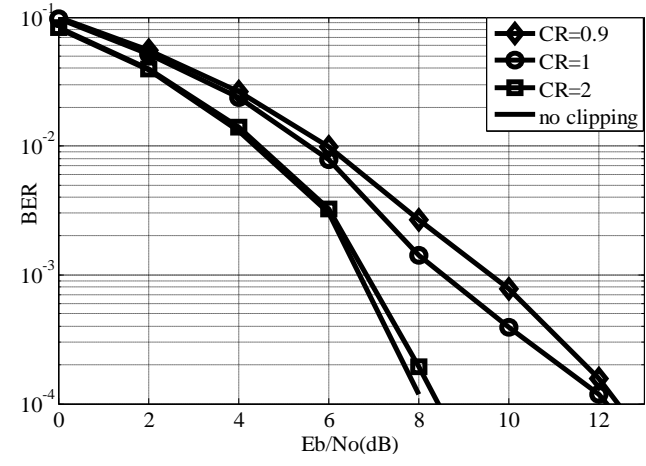

Fig. 4. BER for clipped OFDM ML IDMA

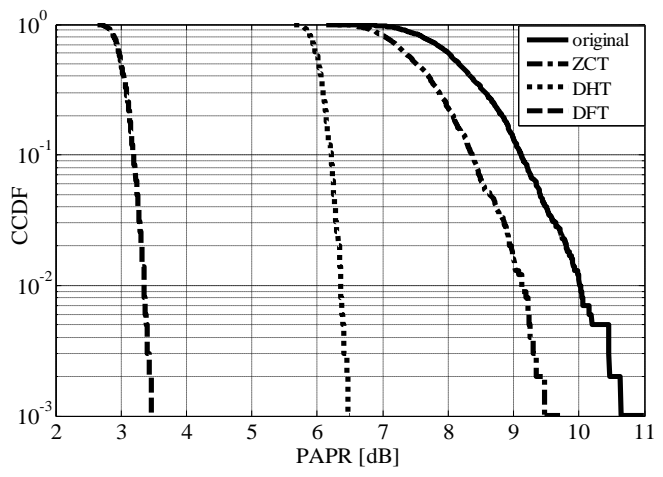

Fig. 5. PAPR of ZCT, DHT and DFT P-OFDM ML IDMA

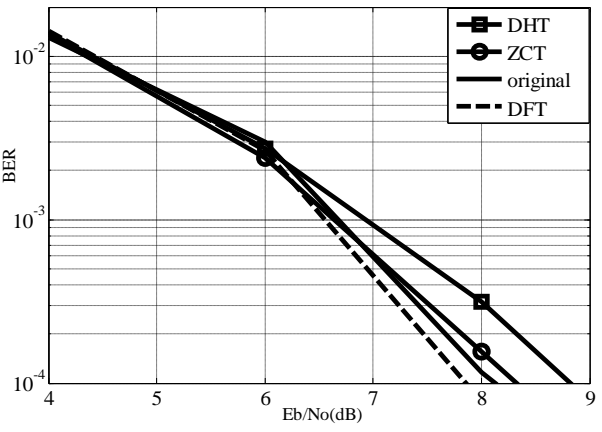

Fig. 6. BER for ZCT, DHT and DFT P-OFDM ML IDMA

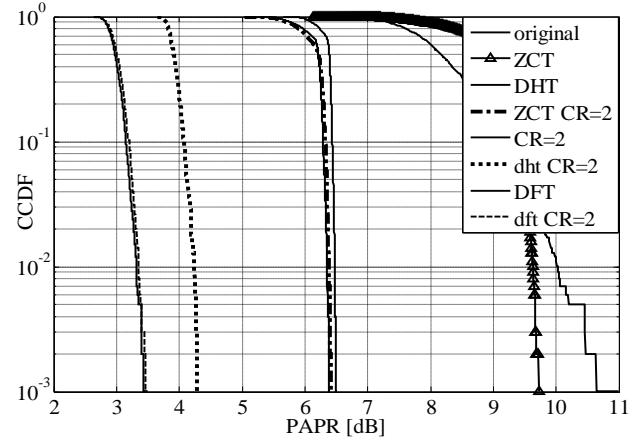

Fig. 7. PAPR of ZCT, DHT and DFT P Clipped systems

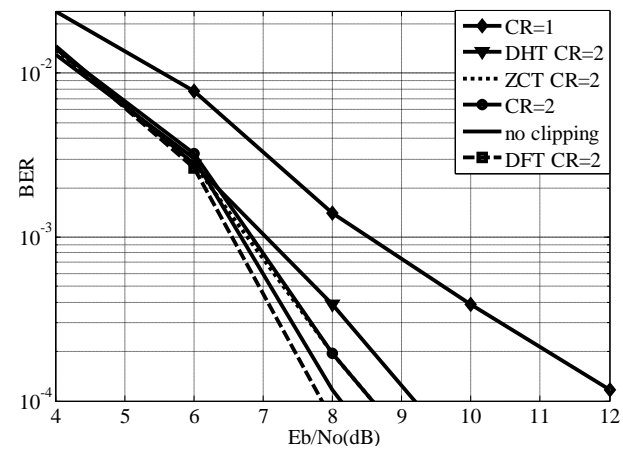

Fig. 8. BER for ZCT, DHT and DFT PC-OFDM ML IDMA

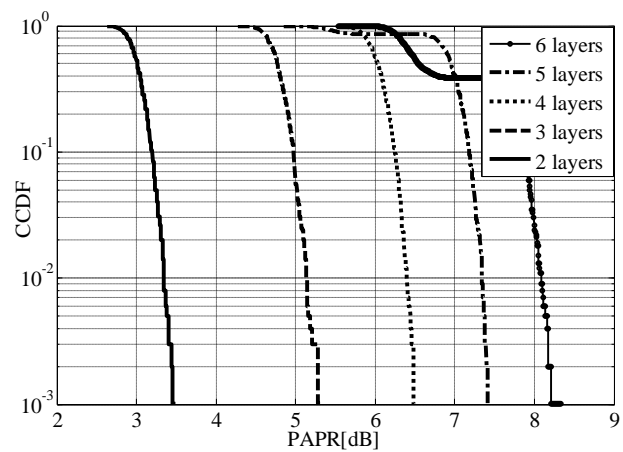

Fig. 9. PAPR DFT P-OFDM ML IDMA with layers $=2,3,4,5$ and 6

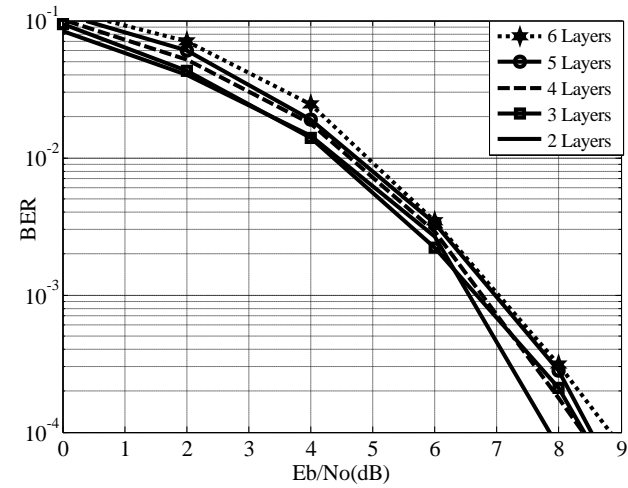

Fig. 10. BER for DFT P-OFDM ML IDMA with layers $=2,3,4,5$ and 6 
TABLE I: PAPR REDUCTION AND BER PERFORMANCE ( $C R=2)$ PAPR AT $=10^{-3}(\mathrm{DB}), \mathrm{EB} / \mathrm{NO}$ AT BER $=10^{-4}\left(\mathrm{DB}^{\prime}\right.$

\begin{tabular}{|l|c|c|}
\hline \multicolumn{1}{|c|}{ Schemes } & PAPR & Eb/No \\
\hline \hline OFDM ML IDMA & 10.9 & 8.1 \\
\hline Clipped OFDM ML IDMA & 6.37 & 8.6 \\
\hline \hline ZCT OFDM ML IDMA & 9.49 & 8.3 \\
\hline ZCT Clipped OFDM ML IDMA & 6.5 & 8.5 \\
\hline \hline DHT OFDM ML IDMA & 6.47 & 8.8 \\
\hline DHT Clipped OFDM ML IDMA & 4.28 & 9.2 \\
\hline \hline DFT OFDM ML IDMA & 3.46 & 7.8 \\
\hline DFT Clipped OFDM ML IDMA & 3.44 & 8.7 \\
\hline
\end{tabular}

TABLE II: ML PAPR AND BER PERFORMANCE $(C R=2)$ PAPR AT $=10^{-3}$ $(\mathrm{DB}), \mathrm{EB} / \mathrm{NO}$ AT BER $=10^{-4}(\mathrm{DB})$

\begin{tabular}{|c|c|c|}
\hline Schemes & PAPR & Eb/No \\
\hline \hline DFT OFDM 2L IDMA & 3.466 & 7.8 \\
\hline DFT OFDM 3L IDMA & 5.246 & 8.4 \\
\hline DFT OFDM 4L IDMA & 6.547 & 8.4 \\
\hline DFT OFDM 5L IDMA & 7.345 & 8.5 \\
\hline DFT OFDM 6L IDMA & 8.214 & 8.8 \\
\hline
\end{tabular}

\section{CONCLUSIONS}

In this paper, PAPR mitigation based on DFT, DHT and ZCT Precoding schemes has been applied to OFDM ML IDMA. CBC iterative receiver is indicated in this paper to recover the data of each layer. Simulation results confirm that the DFT precoder provides better PAPR reduction and improves the BER performance compared with DHT and ZCT precoder with and without clipping. Simulated results for the proposal DFT P OFDM ML IDMA system shows that the proposed system is an efficient bandwidth with increasing the number of layer.

\section{REFERENCES}

[1] T. Peng and M. Ye, "PAPR mitigation in superposition coded modulation systems using selective mapping," in Proc. $3^{\text {rd }}$ International Conference on Computer and Communication Technologies, Phuket, 2012, pp. 103-106.

[2] L. Ping, Q. Guo, and J. Tong, "The OFDM IDMA approach to wireless communication systems," IEEE Wireless Commun. Mag., vol. 14, no. 3, pp. 18-24, Jun. 2007.

[3] M. A. A. Dahab, E. A. Hagras, and A. A. Elhaseeb, "PAPR reduction based on DFT precoding for OFDM signals," International Journal of Future Computer and Communication, vol. 2, no. 4, pp. 325-328, August 2013.

[4] S. H. Han and J. H. Lee, "An overview of peak- to-average power ratio reduction techniques for multicarrier transmission," IEEE Wireless Commun., vol. 12, no. 2, pp. 56-65, Apr. 2005.

[5] T. Jiang and Y. Y. Wu, "An overview: Peak-to- average power ratio reduction techniques for OFDM signals," IEEE Trans. Broadcasting, vol. 54, no. 2, pp. 257-268, Jun. 2008.
[6] R. O'Neill and L. B. Lopes, "Envelope variations and spectral splatter in clipped multicarrier signals," in Proc. $6^{\text {th }}$ Personal, Indoor and Mobile Radio Communications, conference, Toronto, 1995, pp. 71-75.

[7] S. H. Han and J. H. Lee, "PAPR reduction of OFDM signals using a reduced complexity PTS technique," IEEE Sig. Process. Lett., vol. 11, no. 11, pp. 887-890, Nov. 2004.

[8] R. W. Bauml, R. F. H. Fischer, and J. B. Huber, "Reducing the peakto average power ratio of multicarrier modulation by selected mapping," IET Electron. Lett., vol. 32, no. 22, pp. 1257-2056, Oct. 1996.

[9] J. Tong, P. Li, Z. H. Zhang, and W. K. Bhargava, "Iterative soft compensation for OFDM systems with clipping and superposition coded modulation," IEEE Trans. Commun., vol. 58, no. 10, pp. 2861-2870, Oct. 2010.

[10] I. Baig and V. Jeoti, "PAPR analysis of DHT-precoded OFDM system for M-QAM," in Proc. $3^{\text {rd }}$ International Conference on Intelligent and Advanced Systems, Kuala Lumpur, pp. 1-4, 2010.

[11] C. Dageng and W. Yi, "An interleave- division multiplexing (IDM) based modulation for 3GPP LTE downlink," in Proc. $3^{\text {rd }}$ Communications and Networking conference, Beijing, 2008, pp. 474-477.

[12] L. Ping, L. Liu, K. Wu, and W. K. Leung, "Interleave-division multiple access," IEEE Transactions on Wireless Communications, vol. 5, pp. 938-947, Apr. 2006.

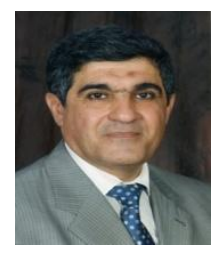

Mohamed A. Aboul-Dahab received the B.S degrees in communication Engineering from Faculty of Engineering, Cairo Univ., M.Sc.and Ph.D. degrees in Communications Engineering from Alexandria Univ., Egypt the is a professor in the Electronics and Communications Engineering department since 1999. $\mathrm{He}$ has been the Head of the Electronics and Computer Engineering Department, College of Engineering and Technology (Alexandria Campus), Arab Academy for Science and Technology and Maritime Transport, AASTMT for 9 years, and the Dean of College of Engineering and Technology (Cairo Campus), AASTMT for 6 years .He has 37 years of teaching experience for the undergraduate students and postgraduate. He has supervised many undergraduate projects and many master and Ph.D. theses in AASTMT and other universities. He is a senior member at the IEEE and its vice chair for Egypt section.

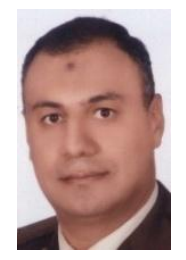

Esam A. A. A. HAGRAS received the B.S. degrees in Electrical Engineering from Faculty of Engineering, Alexandria Univ., Egypt, in 1994, M.S. degrees in Electrical Engineering from Mansoura Univ., Egypt, in 2001, respectively. During 2005-2007, he was on in Dept., of Electrical Engineering, faculty of engineering, Alexandria Univ. In Dec. 2007, he gets the PhD degree in information security in communications. His research interests in the field of information and multimedia security, chaotic cryptography, Hardware implementation of encryption algorithms on FPGA, data compression, digital image watermarking, communication and wireless sensor network security. He has published more than twenty papers on security and communications.

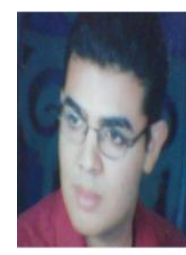

Ahmad A. Elhaseeb received the B.S. degrees in electronics and electrical communication Engineering from Faculty of Engineering, Cairo Univ., Egypt, in 2004, during 2006 - 2009 work in Motorola in GSM networks deployment, during 2009-,now study M.S in Arab Academy for Science, technology and Maritime Transport, Egypt. His research interests in the field of communication. 